\title{
ESTABILIDADE DE COMPOSTOS TIPO HIDROTALCITA Ni-Mg-AI NA REFORMA A VAPOR DO GLP
}

\author{
R. A. R. FERREIRA ${ }^{1}$, J. F. NUNES ${ }^{1}$, P. P. SILVA $^{1}$ e C. E. HORI ${ }^{1}$ \\ ${ }^{1}$ Universidade Federal de Uberlândia, Departamento de Engenharia Química \\ E-mail para contato: cehori@ufu.br
}

\begin{abstract}
RESUMO - Os compostos tipo hidrotalcita podem ser descritos pela fórmula geral: $\left[\mathrm{M}^{2+}{ }_{(1-\mathrm{x})} \mathrm{M}^{3+}{ }_{(\mathrm{x})}(\mathrm{OH})_{2}\right]^{\mathrm{x}+}\left(\mathrm{An}_{-\mathrm{x} / \mathrm{n}}\right) \cdot \mathrm{mH}_{2} \mathrm{O}$ sendo um grupo de materiais de muito interesse na reforma catalítica a vapor de hidrocarbonetos para a produção de hidrogênio. Desta maneira, o objetivo deste trabalho foi avaliar o desempenho de catalisadores tipo hidrotalcita de Ni-Mg-Al (razão molar 3:1) em diferentes concentrações molares na reforma a vapor de GLP (gás liquefeito de petróleo) a $600^{\circ} \mathrm{C}$. Os catalisadores foram caracterizados por DRX mostrando que houve a formação da estrutura lamelar característica da hidrotalcita (HTC), RTP (redução à temperatura programada) e ATG (análise termogravimétrica). $\mathrm{O}$ catalisador $15 \mathrm{NiMg}_{2,55} \mathrm{Al}(15 \%$ de $\mathrm{Ni}$ e 2,55 molar de $\mathrm{Mg})$ foi o que mostrou melhores resultados, apresentando maiores valores para dispersão de $\mathrm{Ni}^{0}$, menor temperatura de redução (RTP) e estabilidade por $24 \mathrm{~h}$ de reação com fração molar para $\mathrm{H}_{2}$ de 0,6 e conversão em torno de $50 \%$ para a mistura, com menor depósito de carbono.
\end{abstract}

\section{INTRODUÇÃO}

Os processos de reforma a vapor para a produção de hidrogênio vêm sendo bastante estudados usando vários hidrocarbonetos como metano, etanol, propano e butano (Ayabe et al., 2003; Shen et al., 2011). O GLP (gás liquefeito de petróleo), que é uma mistura dos gases propano e butano, pode ser uma matéria-prima promissora uma vez que possui grande aplicabilidade como combustível (alto poder calorífico), excelente queima, fácil manuseio, facilidade de armazenamento e transporte e ampla rede de distribuição (Shen et al., 2011; Gokaliler et al., 2008). Contudo, o uso de compostos de cadeias maiores, como é o caso do GLP, na reforma a vapor, gera muito carbono que se deposita na superfície do catalisador, o que pode levar a sua desativação. Assim, a utilização de catalisadores que possam minimizar esses depósitos e apresentar boa estabilidade térmica e maiores dispersões de níquel é de suma importância para o processo de reforma (Laosiripojana et al., 2011).

Os compostos tipo hidrotalcita podem ser descritos pela fórmula geral: $\left[\mathrm{M}_{+(1-x)}^{2} \mathrm{M}^{3+}(\mathrm{x})\right.$ $\left.(\mathrm{OH})_{2}\right]_{\mathrm{x}}+\left(\mathrm{An}_{-\mathrm{x} / \mathrm{n}}\right) \cdot \mathrm{mH}_{2} \mathrm{O}$ (Guil-López et al., 2011) sendo um grupo de materiais de muito interesse na reforma catalítica a vapor de hidrocarbonetos para a produção de hidrogênio (Vaccari, 1999). Estes compostos podem ser sintetizados por diversas técnicas como: co-precipitação e hidrólise de uréia. $\mathrm{O}$ método por co-precipitação é o mais utilizado para a síntese de HTC, podendo ser utilizado de duas formas diferentes: co-precipitação a $\mathrm{pH}$ constante e co-precipitação a $\mathrm{pH}$ variável (Crepaldi et al., 2000). Como o custo de síntese de hidrotalcitas é relativamente baixo, uma grande variedade de 


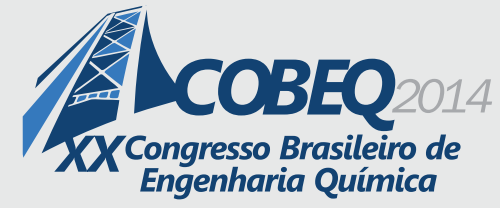

HTCs tem sido sintetizada e aplicada com diversas funções, dentre elas, como catalisadores (Cavani et al., 1991). Assim, o estudo de compostos tipo hidrotalcita (HTC) como precursores catalíticos para processos de reforma vem ganhando espaço, uma vez que apresentam estabilidade térmica e podem levar a boas dispersões de níquel (Vaccari, 1999). Desta maneira, o objetivo deste trabalho foi avaliar o desempenho de catalisadores tipo hidrotalcita de $\mathrm{Ni}-\mathrm{Mg}-\mathrm{Al}$ em diferentes concentrações molares de Ni na reforma a vapor de GLP.

\section{MATERIAIS E MÉTODOS}

\subsection{Síntese em pH constante (co-precipitação)}

As amostras de hidrotalcita foram preparadas pelo método de co-precipitação em $\mathrm{pH}$ constante igual a 10 com diferentes porcentagens em peso de níquel (10 e 15\%) e razões molares entre $\left(\mathrm{Ni}^{2+}+\mathrm{Mg}^{2+}\right) / \mathrm{Al}^{3+}$ fixada em $3: 1$. Uma solução mista de nitratos com $\mathrm{Ni}^{2+} / \mathrm{Mg}^{2+} / \mathrm{Al}^{3+}$ com razões de $10 \mathrm{NiMg}_{2,7} \mathrm{Al}, 15 \mathrm{NiMg}_{2,25} \mathrm{Al}$ e $15 \mathrm{NiMg}_{2,55} \mathrm{Al}$ foram adicionadas em $200 \mathrm{ml}$ de água destilada deionizada com um fluxo constante de $1 \mathrm{ml} \cdot \mathrm{min}^{-1}$ juntamente com uma solução de $\mathrm{NaOH}(2 \mathrm{M})$ à temperatura ambiente, sob agitação vigorosa $(550 \mathrm{rpm})$ mantendo o $\mathrm{pH}$ em 10 . Após o gotejamento, a solução ficou em processo de envelhecimento por 18 horas, sendo filtrada, lavada com água destilada deionizada até $\mathrm{pH}$ 7. O material foi seco em estufa a $105^{\circ} \mathrm{C}$ por 16 horas e macerado. Ao final da preparação, as seguintes amostras foram obtidas: $10 \mathrm{NiMg}_{2,7} \mathrm{Al}(10 \%$ de $\mathrm{Ni}$ e 2,7 molar de $\mathrm{Mg}), 15 \mathrm{NiMg}_{2,25} \mathrm{Al}(15 \%$ de $\mathrm{Ni}$ e 2,25 molar de $\mathrm{Mg})$ e $15 \mathrm{NiMg}_{2,55} \mathrm{Al}$ (15\% de $\mathrm{Ni}$ e 2,55 molar de $\mathrm{Mg}$ ). As amostras foram então submetidas ao processo de calcinação à temperatura de $800^{\circ} \mathrm{C}$ por 5 horas, com fluxo de $30 \mathrm{ml} \cdot \mathrm{min}^{-1}$ de ar sintético, para formação dos óxidos mistos.

\subsection{Difração de Raios X}

As análises de DRX de todos os catalisadores foram realizadas em um equipamento RIGAKU modelo Miniflex, utilizando radiação $\mathrm{CuK \alpha}(1,540 \AA \AA)$. Estas análises foram efetuadas utilizando $2 \Theta$ entre 5 e $70^{\circ}$, um passo de $0,02^{\circ}$ e tempo de contagem de 2 segundos por passo. As fases das HTCs encontradas através do DRX foram analisadas qualitativamente. Para observar as mudanças na estrutura durante o processo de redução, as amostras foram reduzidas em $\mathrm{H}_{2}$ puro com fluxo total de $30 \mathrm{ml} \cdot \mathrm{min}^{-1}$ por duas horas. A seguir, foram passivadas (mistura de $5 \%$ de $\mathrm{O}_{2}$ em argônio a uma vazão de $30 \mathrm{ml} \cdot \mathrm{min}^{-1}$ a temperatura de $1^{\circ} \mathrm{C}$ por $30 \mathrm{~min}$.) e novamente caracterizadas por difração de raios $\mathrm{X}$.

\subsection{Redução à Temperatura Programada - RTP}

As amostras de HTC foram submetidas à redução a temperatura programada (RTP), utilizando uma mistura contendo $2 \%$ de hidrogênio em argônio a uma vazão de $30 \mathrm{ml} \cdot \mathrm{min}^{-1}$. A temperatura foi aumentada gradativamente a uma taxa de $10^{\circ} \mathrm{C} \cdot \mathrm{min}^{-1}$ até atingir a temperatura de $1000^{\circ} \mathrm{C}$. As medidas de RTP foram acompanhadas por um espectrômetro de massas do tipo Quadrupolo marca Balzers. A massa de catalisador utilizada em cada análise foi de aproximadamente $50 \mathrm{mg}$. 


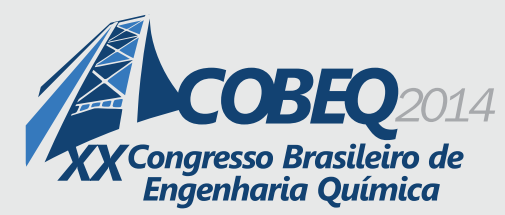

\subsection{Testes catalíticos e Análise Termogravimétrica}

As reações de reforma a vapor de GLP (50\% propano $+50 \%$ butano) foram realizadas com 10 $\mathrm{mg}$ de catalisador/40 $\mathrm{mg}$ de quartzo, uma relação vapor/hidrocarboneto de 7:1, fluxo total da mistura de $200 \mathrm{ml} \cdot \mathrm{min}^{-1}$ a uma temperatura de $600^{\circ} \mathrm{C}$ por um período de 24 horas. O processo de redução foi idêntico ao usado antes da passivação.

As análises termogravimétricas foram realizadas em um analisador diferencial SDT Q600 (TA instruments) acoplado a uma termobalança. As amostras (12mg) foram inicialmente aquecidas na temperatura de $120^{\circ} \mathrm{C}$ em atmosfera de $\mathrm{N}_{2}$ e mantidas nesta temperatura até atingirem massa constante. Em seguida, o $\mathrm{N}_{2}$ foi substituído por uma atmosfera contendo $5 \%$ em volume de $\mathrm{O}_{2}$ e as amostras foram aquecidas até $1000^{\circ} \mathrm{C}$ com uma taxa de $10^{\circ} \mathrm{C} \cdot \mathrm{min}^{-1}$.

\section{RESULTADOS E DISCUSSÃO}

\subsection{Difração de Raios X - DRX}

$\mathrm{Na}$ Figura 1 estão apresentados os difratogramas das amostras de hidrotalcita $10 \mathrm{NiMg}_{2.7} \mathrm{Al}$, $15 \mathrm{NiMg}_{2.25} \mathrm{Al}$ e $15 \mathrm{NiMg}_{2.55} \mathrm{Al}$ sem tratamento (A) e após passivação (B). Os difratogramas mostraram que houve a formação da estrutura da hidrotalcita em todas as amostras, com picos correspondentes em (003) e (006) e os planos não-basais assimétricos em (012), (015) e (018). Sobreposições também foram observadas, embora em reflexões menos intensas em (110) e (113) (Schaper et al., 1989). Após a calcinação e redução das amostras, observou-se a transformação da estrutura formando níquel metálico $\left(2 \theta=44,5 ; 51,8^{\circ}\right)$, óxido de magnésio $\left(2 \theta=36,7 ; 43 ; 62,4^{\circ}\right)$ e a fase espinela $\left(2 \theta=19,3 ; 30,4 ; 36,7 ; 65^{\circ}\right)$ (Li et al., 2010; Li et al., 2011; Shen et al., 2011).
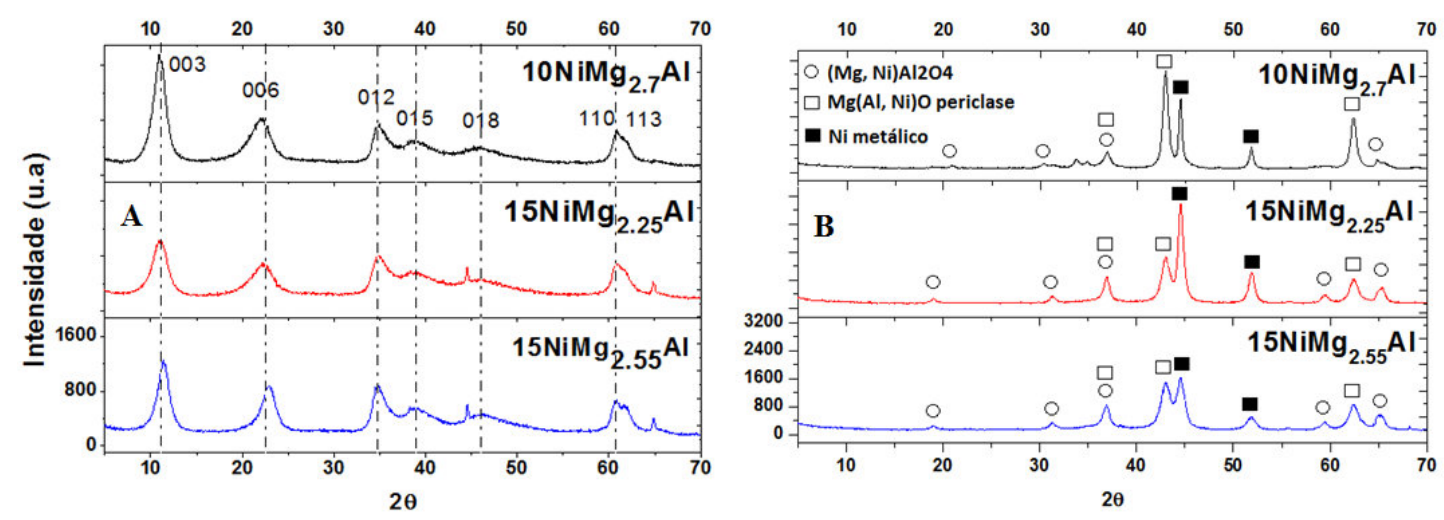

Figura 1 - Difratogramas das amostras sem tratamento (A) e após passivação (B).

Com os dados de DRX das amostras reduzidas e passivadas, utilizando a equação de Scherrer (Equação 1) e a Equação 2 (Parada, 2012) abaixo, foram calculados o diâmetro médio de cristalito $\mathrm{Ni}^{0}$ e sua dispersão na estrutura do catalisador, respectivamente: 


$$
\begin{aligned}
& d_{N i}=\frac{k \cdot \lambda}{\beta \cdot \cos \theta} \\
& \begin{array}{c}
\text { Engenharia Química } \\
\text { Engrasicairo de }
\end{array}
\end{aligned}
$$

Onde a largura a meia altura do pico de difração correspondente a fase $(\boldsymbol{\beta})$, o comprimento de onda da radiação utilizada $(\mathrm{CuK} \alpha-1,540 \AA)$ e a constante que depende do instrumento e do método empregado para o cálculo de $\boldsymbol{\beta}$. Neste caso, $\mathrm{k}=0,9$.

$$
D_{N i}=\frac{3 M W_{N i}}{N_{A} r_{p} \rho_{N i} A_{N i}}
$$

Em que MW corresponde ao peso atômico do níquel (58,6934 g.mol $\left.{ }^{-1}\right), \mathrm{N}_{\mathrm{A}} \mathrm{O}$ número de Avogrado $\left(6,022 * 10^{23}\right), \mathrm{r}_{\mathrm{p}}$ o raio médio de cristalito de níquel metálico (Tabela 1$), \rho_{\mathrm{Ni}}$ a densidade do níquel $\left(8908000 \mathrm{~g} / \mathrm{m}^{3}\right)$ e $\mathrm{A}_{\mathrm{Ni}}$ a área do átomo de níquel metálico $\left(4,831^{*} 10^{-20}\right)$.

A Tabela 1 mostra os valores para o diâmetro médio e a dispersão do $\mathrm{Ni}^{0}$. De acordo com a Tabela 1 foi observado que as amostras apresentaram diâmetros médios de $\mathrm{Ni}^{0}$ muito diferentes, seguindo a ordem inversa do teor de níquel. A amostra que apresentou o maior valor para o diâmetro médio do cristalito foi a $10 \mathrm{NiMg}_{2,7} \mathrm{Al}(21 \mathrm{~nm})$, consequentemente, um menor valor para dispersão (7\%). Esse resultado já era esperado, pois um teor de metal mais alto, geralmente leva menor dispersão metálica (Schulze et al., 2001).

Tabela $1-$ Tamanho médio do cristalito e da dispsersão de $\mathrm{Ni}^{0}$
nos catalisadores
\begin{tabular}{|c|c|c|}
\hline & Diâmetro Ni $(\mathrm{nm})$ & Dispersão $(\%)$ \\
\hline $10 \mathrm{NiMg}_{2.7} \mathrm{Al}$ & 21 & 7 \\
\hline $15 \mathrm{NiMg}_{2.25} \mathrm{Al}$ & 12 & 11 \\
\hline $15 \mathrm{NiMg}_{2.55} \mathrm{Al}$ & 7 & 18 \\
\hline
\end{tabular}

\subsection{Redução à Temperatura Programa - RTP}

Os perfis de RTP das amostras calcinadas a $800^{\circ} \mathrm{C}$, Figura 2 , mostra que as três amostras tiveram somente um pico de redução que pode estar associado à redução de espécies de níquel na solução sólida Mg-Ni-Al-O, o que proporciona uma grande estabilidade aos íons de níquel resultando em altas temperaturas de redução (Schulze et al., 2001; Takehira et al., 2004). O decréscimo da quantidade de magnésio pode influenciar na dispersão do níquel na amostra, proporcionando maiores formações de solução sólida ou a formação do aluminato de níquel (Wang e Lu, 1998). Desta maneira, pode ser observado que, para as amostras com o mesmo teor de níquel (15\%), à medida que a razão $\mathrm{Mg} / \mathrm{Al}$ decresce, ocorre o deslocamento do pico de redução para uma temperatura mais alta, indicando a formação de aluminato de níquel $\left(\mathrm{NiAl}_{2} \mathrm{O}_{4}\right)$, estrutura mais difícil de ser reduzida (Takehira, 2009), concordando com os perfis de RTP apresentados na Figura 2, com temperaturas de 
redução de 890,886 e $874^{\circ} \mathrm{C}$ para as amostras $10 \mathrm{NiMg}_{2,7} \mathrm{Al}, 15 \mathrm{NiMg}_{2,25} \mathrm{Al}$ e $15 \mathrm{NiMg}_{2,55} \mathrm{Al}$, respectivamente.

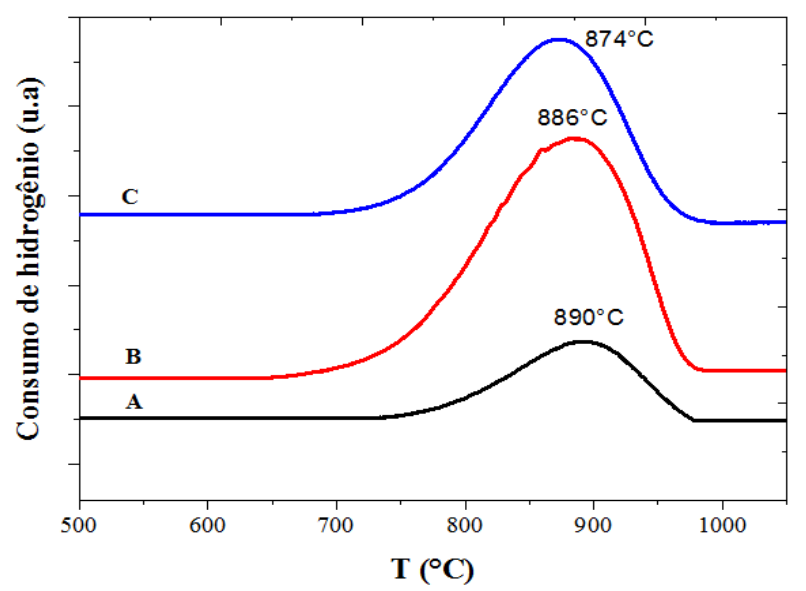

Figura 2 - Perfis de RTP dos catalisadores derivados de hidrotalcita calcinados a $800^{\circ} \mathrm{C}$.

(A) $10 \mathrm{NiMg}_{2,7} \mathrm{Al}$, (B) $15 \mathrm{NiMg}_{2,25} \mathrm{Al}$, (C) $15 \mathrm{NiMg}_{2,55} \mathrm{Al}$.

\subsection{Testes Catalíticos}

A Figura 3 mostra a conversão do GLP e a fração molar de hidrogênio formada em função do tempo de reação de reforma a vapor do GLP. Com o aumento da razão molar $\mathrm{Mg} / \mathrm{Al}$, foi possível observar que a conversão do GLP aumentou cerca de $20 \%$ comparando as hidrotalcitas com $15 \%$ de níquel na estrutura. A presença de uma maior quantidade de níquel e magnésio na estrutura do catalisador $15 \mathrm{NiMg}_{2,55} \mathrm{Al}$, teve dois efeitos que contribuíram para o melhor desempenho dessa amostra. Uma maior dispersão de $\mathrm{Ni}^{0}$, de acordo com a Tabela 1, conferiu ao sistema catalítico maior atividade, pois essa amostra tem um maior número de sítios ativos de níquel expostos. Provavelmente, essa mesma estrutura, que tem um teor mais elevado de magnésio, conferiu uma característica mais básica à hidrotalcita (Schaper et al.; 1989), que por sua vez, contribuiu para menor desativação da amostra durante 24 horas de reação.
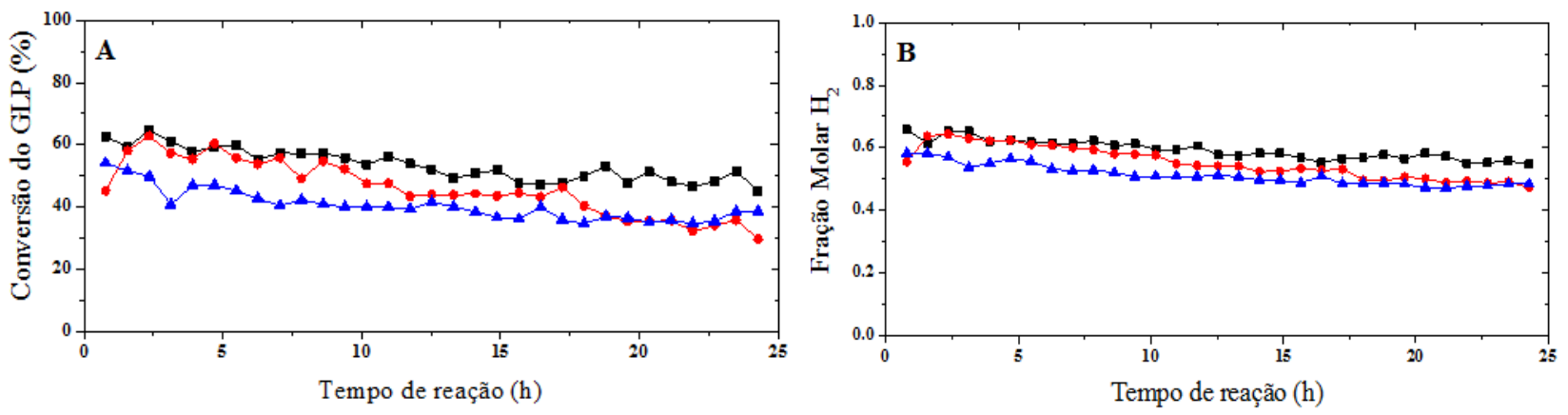

Figura 3 - A - Fração molar de $\mathrm{H}_{2}$ para os catalisadores utilizados; B - Conversão de GLP dos catalisadores para diferentes razões molares Ni/Mg. $(-) 10 \mathrm{NiMg}_{2.7} \mathrm{Al} ;(\bullet-) 15 \mathrm{NiMg}_{2.25} \mathrm{Al}$; $(-)) 15 \mathrm{NiMg}_{2.55} \mathrm{Al}$. 


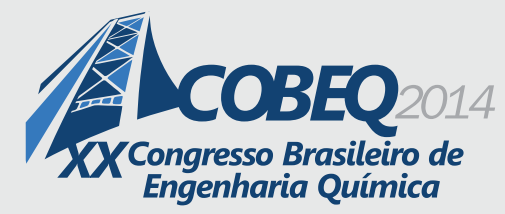

19 a 22 de outubro de 2014

Florianópolis/SC

De acordo com Shen et al. (2011) catalisadores com razões molares de $\mathrm{Mg} / \mathrm{Al}$ maiores, neste caso $15 \mathrm{NiMg}_{2,55} \mathrm{Al}$, apresentaram tamanhos de cristalitos de níquel menores antes da redução e após o processo de pré-reforma (conversão de hidrocarbonetos superiores em metano, hidrogênio e óxidos de carbono a temperaturas baixas) do GLP, resultando em maior estabilidade catalítica, o que concorda com os valores obtidos para dispersão de $\mathrm{Ni}^{0}$ apresentado neste trabalho. Ainda de acordo com estes autores, a diminuição no tamanho dos cristalitos de níquel pode ser responsável pelo processo reversível de redução e oxidação do Ni entre as soluções sólidas de metal e $\mathrm{Mg}-\mathrm{Ni}-\mathrm{O}$ durante a pré-reforma do GLP, justificando uma melhor atividade catalítica e estabilidade. Em relação à fração molar de $\mathrm{H}_{2}$, observou-se que as amostras apresentaram valores próximos em torno de 0,5 com um máximo de 0,6 para o catalisador com 15\% de níquel e 2,55 molar de magnésio.

Ao analisar o RTP (Figura 2) para a amostra com 10\% de níquel, notou-se uma maior temperatura de redução, com possível formação de aluminato de níquel, o que influenciou diretamente em sua menor atividade catalítica conforme pode ser observado nos testes catalíticos, Figura 3. Já em relação as amostras com porcentagem maior de níquel $(15 \%)$, foi observado que à medida que a razão $\mathrm{Mg} / \mathrm{Al}$ diminui, o tamanho médio de cristalito aumenta, diminuindo a dispersão e a atividade catalítica. Uma possível causa para este aumento está relacionado com a diminuição da quantidade dos cátions de $\mathrm{Mg}^{2+}$, com raio iônico de $0,65 \AA$ e ao aumento da quantidade dos cátions de $\mathrm{Al}^{3+}$, que tem raio iônico de $0,50 \AA$, provocando um aumento na densidade dos cátions na lamela, tendo como consequência o aumento da força de atração entre os cátions e os ânions carregados positivamente e negativamente nos espaços interlamelares (Pedrotti, 2010).

Em relação a deposição de coque, as análises de ATG apresentaram valores de 69,70 e $87 \%$ de formação de $\mathrm{C}(\mathrm{s})$ para as amostras $10 \mathrm{NiMg}_{2,7} \mathrm{Al}, 15 \mathrm{NiMg}_{2,55} \mathrm{Al}$ e $15 \mathrm{NiMg}_{2,25} \mathrm{Al}$ após 24 horas de reação, respectivamente. De acordo com a análise térmica diferencial (ATD), pode se observar apenas um pico exotérmico para os três catalisadores com depósito de carbono, entre 580 e $640^{\circ} \mathrm{C}$, temperaturas associadas com a oxidação do carbono filamentoso (Swierczynski et al., 2008). Este tipo de carbono não afeta grandemente a atividade do catalisador, podendo causar a obstrução do reator e queda de pressão após muitas horas de reação, fato não observado nas reações de reforma apresentadas neste trabalho. De acordo com Schulze et al. (2001), independentemente da temperatura de reação, a quantidade de coque formado era sempre maior para os catalisadores com um elevado teor de níquel, teor de magnésio inferior e que também exibem menores dispersões de níquel (Tabela 1), o que pode ser observado para a amostra $15 \mathrm{NiMg}_{2,25} \mathrm{Al}$. Portanto, a maior atividade catalítica foi observada para a amostra $15 \mathrm{NiMg}_{2,55} \mathrm{Al}$ com menor diâmetro de partícula $(7 \mathrm{~nm})$ e maior dispersão $(18,2 \%)$, o que foi comprovado pelo teste catalítico, Figura 3.

\section{CONCLUSÃO}

$\mathrm{O}$ uso de catalisadores tipo hidrotalcita $\mathrm{Ni}-\mathrm{Mg}$-Al pode ser uma boa alternativa para o processo de reforma a vapor de hidrocarbonetos de cadeias maiores e misturas (GLP). O catalisador $15 \mathrm{NiMg}_{2.55} \mathrm{Al}$ apresentou-se como o melhor entre as hidrotalcitas estudadas neste trabalho, com menor tamanho de cristalito de níquel e maior dispersão, concomitante com uma menor temperatura de redução e maiores atividades e estabilidades catalíticas para a produção de 


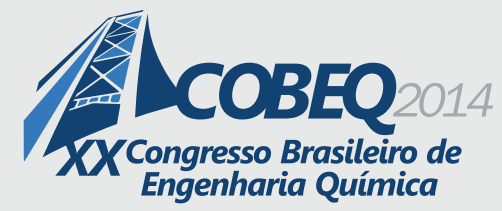

hidrogênio,comprovando a importância da utilização deste tipo de catalisador para a reforma a vapor de hidrocarbonetos de cadeias maiores.

\section{AGRADECIMENTOS}

Os autores agradecem à Fapemig, Capes e CNPq pelo apoio financeiro e à Fapemig (Processo PCE-00089-14) pelo apoio concedido para a vinda nesse congresso.

\section{REFERÊNCIAS}

AYABE, S.; OMOTO, H.; UTAKA, T.; KIKUCHI, R.; SASAKI, K.; TERAOKA, Y.; EGUCHI, K. Catalytic autothermal reforming of methane and propane over supported metal catalysts. Applied Catalysis A: General, v. 241, p. 261-269, 2003.

CAVANI, F.; TRIFIRÒ, F., VACCARI, A., Hydrotalcite-Type Anionic Clays: Preparation, Properties and Applications, Catalysis Today, v. 11, p. 173-301, 1991.

CREPALDI, E. L.; PAVAN, P. C.; VALIM, J. B. Comparative study of the coprecipitation methods for the preparation of Layered Double Hydroxides. Journal of the Brazilian Chemical Society, v.11, p. 64-70, 2000.

GOKALILER, F.; ÇAGLAYAN, B. S.; ONSAN, Z. I.; AKSOYLU, A. E. Hydrogen production by autothermal reforming of LPG for PEM fuel cell applications. International Journal of Hydrogen Energy, v. 33, p. 1383-1391, 2008.

GUIL-LÓPEZ, R.; NAVARRO, R. M.; PENA, M. A.; FIERRO, J. L. G. Hydrogen production by oxidative ethanol reforming on $\mathrm{Co}, \mathrm{Ni}$ and $\mathrm{Cu}$ ex-hydrotalcite catalysts. International Journal of Hydrogen Energy, v. 36, p. 1512-1523, 2011.

LAOSIRIPOJANA, N.; SUTTHISRIPOK, W.; CHAROJROCHKUL, S.; ASSABUMRUNGRAT, S. Steam reforming of LPG over $\mathrm{Ni}$ and $\mathrm{Rh}$ supported on $\mathrm{Gd}-\mathrm{CeO}_{2}$ and $\mathrm{Al}_{2} \mathrm{O}_{3}$ : Effect of support and feed composition. Fuel, v. 90, p. 136-141, 2011.

LI, M.; WANG, X.; LI, S.; WANG, S.; MA, X. Hydrogen production from ethanol steam reforming over nickel based catalyst derived from $\mathrm{Ni} / \mathrm{Mg} / \mathrm{Al}$ hydrotalcite-like compounds. International Journal of Hydrogen Energy, v. 35, p. 6699-6708, 2010.

LI, Z.; SONG, Y.; WANG, J.; LIU, Q.; YANG, P.; ZHANG, M. "Study of Structural Transformations and Phases Formation upon Calcination of Zn-Ni-Al Hydrotalcite Nanosheets”. Bulletin of Materials Science, v. 34, n. 2, p. 183-189, 2011.

PARADA, HECTOR ENRIQUE ROJAS. Produção de Hidrogênio a partir da reforma de biogás usando perovskitas a base de Lantânio e Níquel dopados com Estrôncio, Dissertação de Mestrado, Universidade Federal de Uberlândia, 2012. 
PEDROTTI, W. Hidrotalcitas de Ni-Mg-Al como precursores de catalisadores para produção de gás de síntese a partir de biogás. Dissertação de Mestrado, Universidade Federal de São Carlos, 2010.

SCHAPER, H.; BERG-SLOT, J. J.; STORK, W. H. J. Stabilized Magnesia: A Novel Catalyst (Support) Material. Applied Catalysis, v. 54, n. 1, p. 79-90, 1989.

SCHULZE, K.; MAKOWSKI, W.; CHYZY, R. D.; GEISMAR, G. Nickel doped hydrotalcite as catalyst precursors for the partial oxidation of light paraffins. Applied Clay Science, v. 18, p. 59-69, 2001.

SHEN, K.; WANG, X.; ZOU, X.; WANG, X.; LU, X.; DING, W. Pre-reforming of liquefied petrolum gas over nickel catatysts supported on magnesium aluminum mixed oxides. International Journal of Hydrogen Energy, v. 36, p. 4908-4916, 2011.

SWIERCZYNSKI, D.; COURSON, C., KIENNEMANN A. Study of steam reforming of toluene used as model compound of tar produced by biomass gasification. Chem. Eng. Process, v. 47, p. 508-513, 2008.

TAKEHIRA, K. Intelligent reforming catalysts: Trace noble metal-doped $\mathrm{Ni} / \mathrm{Mg}(\mathrm{Al}) \mathrm{O}$ derived from hydrotalcites. Journal of Natural Gas Chemistry, v. 18, p. 237-259, 2009.

TAKEHIRA, K., SHISHIDO, T., WANG, P., KOSAKA, T., TAKAKI, K. Autothermal reforming of $\mathrm{CH} 4$ over supported ni catalysts prepared from $\mathrm{Mg}$-Al hydrotalcite -like anionic clay. Journal of Catalysis, v. 221, p. 43-54, 2004.

VACCARI, A. Clays and catalysis: a promising future. Applied Clay Science, v. 14, p. 161-198, 1999.

WANG, S.; LU, G. Q. Role of $\mathrm{CeO} 2$ in $\mathrm{Ni} / \mathrm{CeO} 2-\mathrm{A} 12 \mathrm{O} 3$ catalysts for carbon dioxide reforming of methane. Appl. Catal. B, v. 19, p. 267-277, 1998. 